\title{
Factores que intervienen en la no prescripción de betabloqueantes en los pacientes con infarto agudo de miocardio
}

\author{
J. SALAS CORONAS, A. MONTIEL TRUJILLO, A. LÓPEZ MARTÍNEZ, \\ J. L. ZAMBRANA GARCÍA, M. DELGADO FERNÁNDEZ, F. DÍEZ GARCÍA
}

Hospital de Poniente. Servicio de Medicina Interna. El Ejido, Almería

\author{
FACTORS THAT INFUENCE THE NON PRESCRIPTION \\ OF BETA-BLOCKERS IN PATIENTS WITH ACUTE MYOCARDIAL \\ INFARCTION
}

\section{RESUMEN}

Objetivo: Estudiar los factores que intervienen en la no prescripción de fármacos betabloqueantes en los pacientes que son dados de alta con diagnóstico de infarto agudo de miocardio (IAM).

Método: Estudio retrospectivo de los pacientes dados de alta de nuestro servicio con diagnóstico principal de IAM durante el año 1998. Se consideraron como variables la edad, sexo, diabetes mellitus, presencia de arteriopatía periférica, la función ventricular y la condición de EPOC.

Resultados: Fueron incluidos 60 pacientes con IAM, 18 de los cuales (30\%) fueron dados de alta sin betabloqueantes. Los pacientes a los que no se prescribió betabloqueantes presentaban una edad media de 77 años frente a los 60 años de media del grupo que se dio de alta con estos fármacos $(p<0,0001)$. Asimismo, la disfunción ventricular $(\mathrm{p}<0,031)$ y el sexo femenino $(p<0,016)$ condicionaron también un menor uso de estos fármacos. En el análisis de regresión multivariable se observa que la edad es el principal predictor para el uso de betabloqueantes $(\mathrm{p}<0,0001)$.

Conclusiones: La edad es el principal factor que condiciona la no prescripción de betabloqueantes en los pacientes con IAM en nuestro Servicio. A pesar de la potencialidad de efectos adversos de estos fármacos en la población de mayor edad, los datos obtenidos hasta el momento muestran un claro beneficio en los subgrupos de riesgo (edad avanzada, insuficiencia cardiaca,...), por lo que debiera extenderse su uso a este grupo de población a no ser que existieran contraindicaciones absolutas.

PALABRAS CLAVE: Infarto agudo de miocardio. Betabloqueantes. Sexo. Edad. Disfunción de ventrículo izquierdo.

\section{ABSTRACT}

Objective: To study the factors that influence the non prescription of beta-blockers in patients discharged with a diagnosis of acute myocar dial infarction (AMI).

Method: A retrospective study was done of all patients discharged from our Service in the year 1998, with a diagnosis of AMI. The variables considered were age, sex, diabetes mellitus, peripheral vascular disease, left ventricle dysfunction and COPD.

Results: 60 patients with AMI were included in the study, 18 of whom (30\%) were discharged without beta-blockers. The average age of these patients was 77 years, while the average age of those discharged with these pharmaceutical agents was 60 years $(p<0.0001)$. Likewise, left ventricle dysfunction $(p<0.031)$ and female gender $(p<0.016)$, also negatively influenced the use of these drugs. It was observed with multi variable regression analysis that age was the main predictor for the use of beta-blockers $(p<0.0001)$.

Conclusion: Age is the main factor that influences the non prescrip tion of beta-blockers in patients with AMI in our Service. In spite of the potential adverse effects of the drugs in the advanced age population, the data so far obtained demonstrates a clear benefit in the subgroups at risk (advanced age, heart failure,...). Therefore it's use should be exten ded to this group of population as long as there are no absolute contrain dications.

KEY WORDS: Acute myocardial infarction. Beta-blockers. Sex. Age. Left ventricle dysfunction.

Salas Coronas J, Montiel Trujillo A, López Martínez A, Zambrana García JL, Delgado Fernández M, Díez García F. Factores que intervienen en la no prescripción de betabloqueantes en los pacientes con infarto agudo de miocardio. An Med Interna (Madrid) 2001; 18: 187-190.

\section{INTRODUCCIÓN}

Los betabloqueantes (BB) son un grupo de fármacos que actúa produciendo un bloqueo competitivo y reversible de las respuestas de las catecolaminas mediadas a través de la estimulación de los receptores beta-adrenérgicos. Entre sus acciones sobre el miocardio, destacan un efecto inotrópico negativo, con reducción del estrés parietal y la demanda miocárdica de oxígeno; un efecto cronotrópico negativo, mejorando la perfusión coronaria; la reducción del desarrollo de arritmias al actuar sobre el balance entre el sistema simpático y parasimpático, y la actuación sobre factores que intervienen en la dilatación del ventrículo izquierdo.

Diversos estudios han demostrado que la administración precoz de betabloqueantes y su mantenimiento a largo plazo en los pacientes que han sufrido un infarto agudo de miocar-

Trabajo aceptado: 4 de Septiembre de 2000

Correspondencia: Joaquín Salas Coronas. C/ Limonar nº 1, 11-D. 04720 Aguadulce. Almería. 
dio (IAM) mejora su supervivencia $(1,2)$. En la actualidad, está aceptado que los BB deberían ser un componente integral en el manejo médico estándar del IAM (3). Sin embargo, debido a sus potenciales efectos secundarios, son en muchas ocasiones infrautilizados en el tratamiento de esta patología (4), fundamentalmente en aquellos pacientes que se pueden considerar como grupos de riesgo (edad avanzada, disfunción de ventrículo izquierdo, enfermedad pulmonar...), cuando es precisamente en dichos grupos en los que se observa un mayor beneficio con su uso $(5,6)$. Es por ello que algunas organizaciones consideran el uso de la terapia betabloqueante después del IAM como un indicador de buena calidad en los cuidados médicos (7).

El objetivo de nuestro trabajo es analizar los factores que intervienen en la no prescripción de fármacos betabloqueantes en los pacientes que son dados de alta de nuestro servicio con diagnóstico de infarto agudo de miocardio, lo que nos permitirá además obtener un parámetro de la calidad de nuestra asistencia a este grupo de población.

\section{MATERIAL Y MÉTODOS}

Hemos realizado un estudio retrospectivo de los pacientes dados de alta de nuestro servicio en el año 1998 con diagnóstico principal de infarto agudo de miocardio. Se emplearon los informes médicos de alta y las historias clínicas de los pacientes. En total fueron incluidos 60 pacientes, siendo el $20 \%$ mujeres $(n=12)$. La edad media fue de 65,4 años (37-91). Para analizar la prescripción de betabloqueantes se consideraron como variables la edad, sexo, diabetes mellitus, la presencia de arteriopatía periférica, la función ventricular obtenida mediante ecocardiografía y la existencia de enfermedad pulmonar obstructiva crónica (EPOC). Para el análisis estadístico se empleó el programa CSS (Statsof, USA). Se consideraron significativos los valores de $\mathrm{p}$ menores de 0,05 .

\section{RESULTADOS}

La tabla I muestra la distribución de las variables en función de la prescripción o no de betabloqueantes. De los 60 pacientes, 18 (30\%) fueron dados de alta sin BB. Los fármacos usados fueron atenolol (39 pacientes, con una dosis media de $62 \mathrm{mg} /$ día), propanolol ( 2 pacientes con dosis media de 27 $\mathrm{mg}$ /día), y carvedilol (1 paciente con $12,5 \mathrm{mg} /$ día).
Los pacientes a los que no se prescribió BB presentaban una edad media de 77 años frente a los 60 años del grupo que se dio de alta con estos fármacos $(\mathrm{p}<0,0001)$ (Fig. 1). La disfunción ventricular condicionó también un menor uso de BB (p<0,031) (Fig. 2). En cuanto al sexo, observamos como la prescripción de betabloqueantes en las mujeres fue sensiblemente inferior que en los varones $(\mathrm{p}<0,016)$ (Fig. 3), aunque este hecho puede deberse en parte a la superior edad media que muestran las mujeres (73 años) frente a los hombres $(63,7$ años) ( $\mathrm{p}<0,045)$. No observamos diferencias significativas en relación con la condición de EPOC, la presencia de arteriopatía periférica o la existencia de diabetes mellitus, quizás en los dos primeros casos por el escaso número de pacientes encontrados.

La tabla II muestra el análisis de regresión múltiple, en el que se emplearon como variables independientes las referidas anteriormente. Este análisis demostró que, en la población objeto de nuestro estudio, la edad predijo la prescripción de betabloqueantes al alta en los pacientes con diagnóstico de IAM $(\mathrm{p}<0,0001)$.

\section{DISCUSIÓN}

La edad es el principal factor que ha condicionado la no prescripción de betabloqueantes en los pacientes con infarto agudo de miocardio en nuestro Servicio. La presencia de disfunción ventricular y el sexo femenino también influyeron, aunque en menor medida, en el menor uso de estos fármacos en dichos pacientes.

Diversos estudios han demostrado que la administración de BB a los pacientes que han sufrido un infarto agudo de miocardio mejora su supervivencia a largo plazo $(1,2)$. Este efecto beneficioso es incluso superior en aquellos pacientes con más factores de riesgo para recibir estos fármacos, como la presencia de insuficiencia cardiaca, la superior edad o la existencia de una enfermedad pulmonar asociada (5-6). A pesar de todas estas evidencias, el uso de BB está aún por debajo de lo que debiera considerarse como un nivel óptimo. Gottlieb y cols. (8), analizando más de 200.000 pacientes dados de alta con IAM y recogidos en el Cooperative Cardiovascular Project, observa que sólo en el 34\% de los mismos se prescribieron BB al alta. Sin embargo, estos pacientes presentaron una mortalidad un $40 \%$ más baja que aquellos que se fueron de alta sin BB. Esta reducción de la mortalidad fue incluso superior que la encontrada en un metaanálisis de 23

\section{TABLA I}

DISTRIBUCIÓ N DE LAS VARIABLES ESTUDIADAS EN FUNCIÓN DE LA PRESCRIPCIÓN O NO DE BETABLOQUEANTES

\begin{tabular}{llcc}
\hline Variable & Con betabloqueantes $N=42$ & Sin betabloqueantes $N=18$ & Nivel de $\mathrm{p}^{*}$ \\
\hline Edad (años) 1 & $60(12)$ & $77(8.8)$ & 0,0001 \\
Sexo & 37 varones, 5 mujeres & 11 varones, 7 mujeres & 0,016 \\
Diabetes mellitus & 14 & 9 & 0,231 \\
Disfunción de VI & 7 & 8 & 0,031 \\
Arteriopatía. & 3 & 2 & 0,617 \\
EPOC & 0 & 2 & - \\
\hline
\end{tabular}

1 media (DE). * t de Student.

VI: ventrículo izquierdo; EPO C: enfermedad pulmonar obstructiva crónica. 


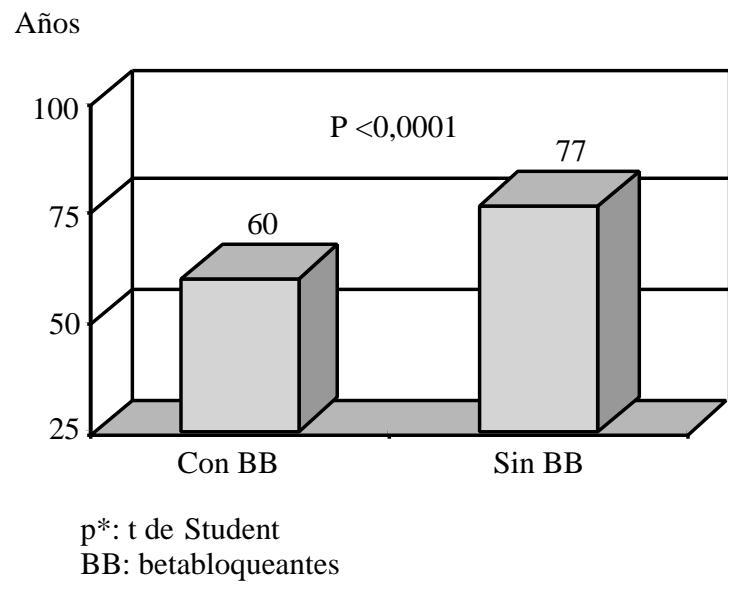

Fig. 1. Edad media de los pacientes dados de alta con y sin betabloqueantes.

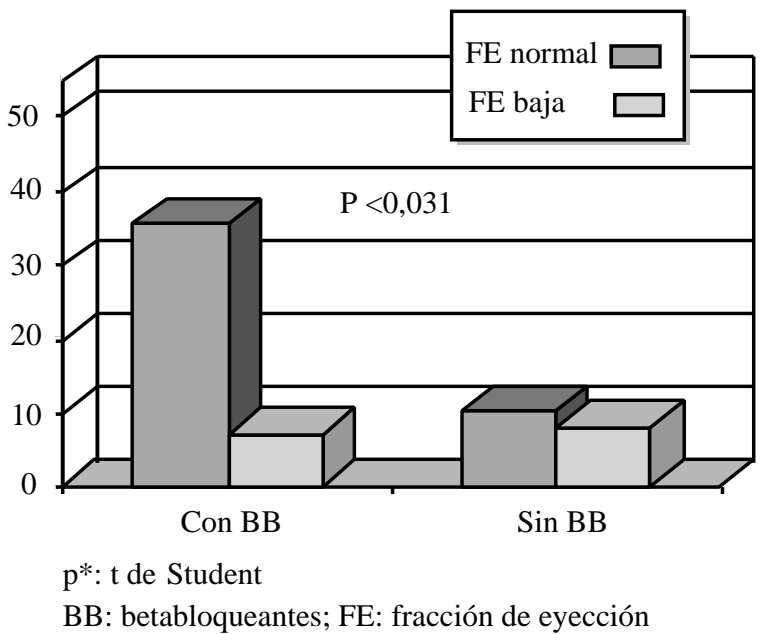

Fig. 2. Prescripción de betabloqueantes en función de la existencia o no de disfunción ventricular.

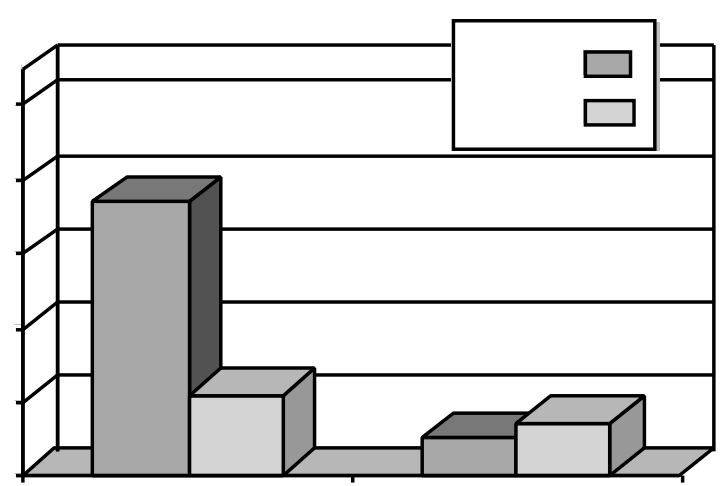

Fig. 3. Prescripción de betabloqueantes en función del sexo.

estudios (9), que fue del $22 \%$. Otros trabajos, como el realizado en hospitales suizos (10), elevan al $51 \%$ el número de pacientes tratados al alta con estos fármacos. La prescripción de $\mathrm{BB}$ en un $70 \%$ de los pacientes incluidos en nuestro estudio
TABLA ॥

ANÁLISIS DE REGRESIÓN M ÚLTIPLE, INDICANDO

LAS VARIABLES Q UE ENTRAN EN LA ECUACIÓN

\begin{tabular}{lllll}
\hline Variables & M últiple R & Múltiple $R^{2}$ & Cambio R $^{2}$ & Nivel $p$ \\
\hline Edad & 0,52197 & 0,27245 & 0,27245 & 0,00008 \\
EPOC & 0,56445 & 0,31860 & 0,04615 & 0,07486 \\
Sexo & 0,59985 & 0,35982 & 0,04121 & 0,08526 \\
Disfunción de VI & 0,62682 & 0,39291 & 0,03308 & 0,11618
\end{tabular}

EPOC: enfermedad pulmonar obstructiva crónica. VI: ventrículo izquierdo.

creemos que es reflejo de una buena calidad asistencial en los sujetos afectos de esta patología.

En cualquier caso, la utilización de BB está muy condicionada por diversos factores. La edad es quizás el principal de ellos. En nuestro estudio, la edad media de los pacientes que fueron dados de alta sin BB era de 77 años frente a los 60 años de media del grupo que se dio de alta con estos fármacos. Este hecho es corroborado por diversos estudios. Malaterre y cols. (11) observan cómo sólo se ha prescrito BB al $2 \%$ de los pacientes mayores de 80 años con infarto agudo de miocardio, y paradójicamente, más del $40 \%$ eran dados de alta con antagonistas del calcio, fármacos que no han demostrado un aumento de la supervivencia en el IAM. Brandt y cols. (12) observan, en 369 pacientes con IAM estudiados consecutivamente, como los BB fueron prescritos al alta en el $77 \%$ de los sujetos más jóvenes frente sólo al $27 \%$ de los pacientes de mayor edad. En un estudio italiano (13), el 56\% de los pacientes menores de 60 años recibían esta terapia frente al $25 \%$ de los que tenían una edad entre 70 y 79 años. Sin embargo, el beneficio de estos fármacos en los pacientes de mayor edad es claro, llegando a reducir la mortalidad hasta en un 32\% (8).

El sexo es otro factor que condiciona el uso de BB. Hemos visto una menor prescripción al alta de forma significativa en mujeres, sin que en este grupo de población fueran diferentes el resto de factores que pudieran influir en dicho uso, salvo la edad, que fue sensiblemente superior. Diversos estudios (14) han demostrado una tendencia a infrautilizar este tipo de fármacos, que podría explicarse en parte por la edad superior que tienen las mujeres con infarto agudo de miocardio respecto a los hombres. Pero la realidad es que las mujeres tienen mayores tasas de mortalidad por infarto agudo de miocardio incluso controlando la variable edad (15), y que es atribuible en parte a que reciben con menos frecuencia tratamiento con aspirina, heparina o BB. Cuando se emplea terapéutica trombolítica, las mujeres son tratadas una media de 14 minutos más tarde y experimentan un mayor número de eventos hemorrágicos mayores. Además, la cateterización cardiaca, procedimientos de angioplastia coronaria transluminal percutánea y la cirugía de bypass son usadas con menos frecuencia en mujeres. Algunos autores han sugerido que las mayores tasas de mortalidad operatoria en las mujeres sometidas a cirugía de bypass podrían ser explicadas en parte porque las mujeres consideradas para este tratamiento presentan una enfermedad coronaria más avanzada $(16,17)$. Por todo ello, varios estudios (18-20) han demostrado un peor pronóstico en las mujeres que sufren un IAM respecto a los hombres.

La disfunción ventricular ha sido el tercer factor que ha influido significativamente en nuestro estudio en la no pres- 
cripción de BB. Tras el concepto inicial de que el efecto inotrópico negativo supondría un detrimento en los pacientes con fallo cardiaco congestivo, recientes estudios $(21,22)$ han demostrado que el uso a largo plazo de los BB en estos pacientes es beneficioso. En términos de reducción de mortalidad, esta ha experimentado una reducción del $40 \%$ en los pacientes con fallo cardiaco que recibieron $\mathrm{BB}$ respecto a los que no los recibieron (8). El riesgo relativo de muerte disminuyó un $40 \%$ en los pacientes con fracción de eyección del ventrículo izquierdo entre el 20 y el $49 \%$, y en un $32 \%$ en los pacientes con una fracción de eyección inferior al $20 \%$. Sin embargo, como los pacientes con disfunción ventricular tienen unas tasas de mortalidad más elevadas, el beneficio absoluto para este grupo es superior.

No hemos observado diferencias significativas en cuanto a la utilización de BB en los pacientes con diabetes mellitus, EPOC o arteriopatía periférica, probablemente en los últimos dos casos debido al escaso número de pacientes encontrados en nuestro estudio. Sin embargo, estas patologías han sido consideradas clásicamente como contraindicaciones, al menos relati- vas, para el uso de estos fármacos. Los BB pueden empeorar el control metabólico en la diabetes mellitus y enmascarar los síntomas de hipoglucemia. Sin embargo, se han observado cifras de reducción de la mortalidad en pacientes diabéticos de hasta el $36 \%$ cuando se usan BB tras el IAM $(8,23)$. Además, dado que la mortalidad tras el IAM es superior en los diabéticos (24), el beneficio en términos de supervivencia es aún mayor. En cuanto a la presencia de enfermedad pulmonar, el efecto broncoconstrictor de los BB han limitado su utilización en estos pacientes a pesar de los datos que demuestran un importante efecto sobre la supervivencia en este grupo de sujetos (8).

En conclusión, podemos decir que la edad es el principal factor que condiciona la no prescripción de betabloqueantes en los pacientes con IAM en nuestro Servicio. A pesar de la potencialidad de efectos adversos de estos fármacos en la población de mayor edad, los datos obtenidos hasta el momento muestran un claro beneficio en los subgrupos de riesgo (edad avanzada, insuficiencia cardiaca, diabetes mellitus,...), por lo que debiera extenderse su uso a este grupo de población a no ser que existieran contraindicaciones absolutas.

\section{Bibliografía}

1. The Norwegian Multicenter Study Group. Timolol-induced reduction in mortality and reinfarction in patients surviving acute myocardical infarction. N Engl J Med 1981; 304: 801-7.

2. Yusuf S, Peto R, Lewis J, Collins R, Sleight P. Beta-blockade during and after myocardical infarction: An overview of the randomized trials. Prog Cardiovasc Dis 1985; 27: 335-71.

3. ACC/AHA Guidelines for the management of patients with acute myocardical infarction. J Am Coll Cardiol 1996; 28: 1328-428.

4. Brand DA, Newcomer LN, Freiburger A, Tian H. Cardiologists' practices compared with practice guidelines: use of beta-blockade after acute myocardical infarction. J Am Coll Cardiol 1995; 26: 1432-6.

5. ISIS-1 (First International Study of Infarct Survival). Collaborative Group: Randomized trial of intravenous atenolol among 16027 cases of suspected acute myocardical infarction: ISIS-1. Lancet 1986; 1: 57-66.

6. MIAMI: Metoprolol in Acute Myocardical Infarction. Am J Cardiol 1985; 56: 10G-14G.

7. Ellerbeck EF, Jencks SF, Radford MJ, Kresowik TF, Graig AS, Gold JA et al. Quality of care for Medicare patients with acute myocardical infarction: a four-state pilot study from the Cooperative Cardiovascular Project. JAMA 1995; 273: 1509-14.

8. Gottlieb S, McCarter R, Vogel R. Effect of beta-blockade on mortality among high-risk and low-risk patients after myocardical infarction. N Engl J Med 1998; 339: 489-97.

9. Yusuf S, Wittes J, Friedman L. Overview of results of randomized clinical trials in heart disease. I. Treatments following myocardical infarction. JAMA 1988; 260: 2088-93.

10. Naegeli B, Bertel O, Urban P, Angehrn W, Siegrist P, Stauffer J, et al. Acute myocardial infarct in Switzerland: results from the PIMICS Heart Infarct Register. PIMICS Project. Schweiz Med Wochenschr 1998; 128: 729-36.

11. Malaterre HR, Daver L, Deharo JC, Bory M, Djiane P. Myocardical infarction after 80 years of age. Apropos of 157 cases. Arch Mal Coeur Vaiss 1994; 87: 875-81.

12. Brandt CM, Wees-Ponchon A, Nisand G, Verdun A, Jobard P, Attali P, et al. Survival in acute myocardial infarction in a group of 369 patients consecutively admitted between 1988 and 1992. Analysis of risk factors and medical procedure. Arch Mal Coeur Vaiss 1994; 87: 861-8.
13. Garini A, Emanuelli C, Fadin BM, Manzetti G, Distante R, Astorri E, et al. The effect of age on early and late mortality after an acute myocardial infarct. Minerva Cardioangiol 1994; 42: 259-68.

14. Nohria A, Vaccarino V, Krumholz HM. Gender differences in mortality after myocardial infarction. Cardiol Clin 1998; 16: 45-57.

15. Chandra NC, Ziegelstein RC, Rogers WJ, Tiefenbrunn AJ, Gore JM, French WJ et al. Observations of the treatment of women in the United States with myocardial infarction. Arch Intern Med 1998; 158: 981-988.

16. Khan SS, Nessim S, Gray R, Czer L, Chaux A, Matloff J. Increased mortality of women in coronary artery bypass surgery: evidence for referral bias. Ann Intern Med 1990; 112: 561-67.

17. Mark DB, Shaw LK, DeLong ER, Califf RM, Pryor DB. Absence of sex bias in the referral of patients for cardiac catheterization. N Engl J Med 1994; 330: 1101-106.

18. Puletti M, Sunseri L, Curione M, Erba SM, Borgia C. Acute myocardial infarction: sex-related differences in prognosis. Am Heart J 1984; 108: 63-66.

19. Tofler GH, Stone PH, Muller JE, Willich SN, Davis VG, Poole WK et al, and the MILIS Study Group. Effect of sex and race on prognosis after myocardial infarction: adverse prognosis for women, particulary black women. J Am Coll Cardiol 1987; 9: 473-482.

20. Weaver WD, White HD, Wilcox RJ, Aylward PE, Morris D, Guerci A et al. for the GUSTO-I investigators. Comparisons of characteristics and outcomes among women and men with acute myocardial infarction treated with thrombolytic therapy. JAMA 1996; 275: 777-782.

21. Packer M, Bristow MR, Cohn JN, Colucci WS, Fowler MB, Gilbert EM et al. The effect of carvedilol on morbidity and mortality in patients with chronic heart failure. N Engl J Med 1996; 334: 1349-55.

22. Waagstein F, Bristow MR, Swedberg K, Camerini F, Fowler MB, Silver MA et al. Beneficial effects of metoprolol in idiopathic dilated cardiomyopathy. Lancet 1993; 342: 1441-6.

23. Gundersen T, Kjekshus J. Timolol treatment after myocardial infarction in diabetic patients. Diabetes Care 1983; 6: 285-90.

24. Zuanetti G, Latini R, Maggioni AP, Santoro L, Franzosi MG. Influence of diabetes on mortality in acute myocardial infarction: data from the GISI-2 study. J Am Coll Cardiol 1993; 22: 1788-94. 